\title{
Image Retrieval using Classification based on Color
}

\author{
Harsh Kumar Sarohi \\ Department of Computer Science \\ Amity University \\ Noida, India
}

\author{
Farhat Ullah Khan \\ Department of Computer Science \\ Amity University \\ Noida, India
}

\begin{abstract}
This paper proposes a new color classification based approach for Image Retrieval. Information in form of images is increasing at a very fast rate therefore there is need for faster and effective retrieval of specific image from image repositories. Content Based Image Retrieval (CBIR) is considered most efficient to search large image collections. The proposed method uses two color spaces to form two feature vectors and then uses Euclidean distance to categorize images on the basis of color and which limits the search to specific category. This results in speedy retrieval. For reducing computational time only sum of RGB component is used for retrieving the specified image.
\end{abstract}

\section{General Terms}

Image Retrieval, Image Retrieval based on Color.

\section{Keywords}

Content Based Image Retrieval (CBIR), Query By Image Content (QBIC), Image Retrieval Using Color Classification, Categorizing Using Euclidean Distance.

\section{INTRODUCTION}

In order to deal with searching in large image datasets two solutions are available

\subsection{Image annotation}

In this approach images are tagged and meta-data is attached to images [1], then keywords are used in search interface for getting specific image. However, tagging of images is time consuming, leads to inefficient data storage and since every image cannot be described in the form of keywords there is too much responsibility on the end user for forming the query. The solution to problem encountered is (Content Based Image Retrieval) CBIR.

\subsection{Content Based Image Retrieval}

In this technique images are retrieved based on content of an image. Two major steps in CBIR are

\subsubsection{Feature Extraction Algorithm}

This algorithm processes the image and compute feature vector. This feature vector [2] is used for the extraction of information from data set. Features can be human vision related or low-level features, objects related or middle-level features, high-level features or semantic related. Most commonly used features for retrieval are color, shape, texture.

\subsubsection{Matching Algorithm}

It performs comparison between extracted features of images to check whether they are similar or not and up to what extent they match. It uses distance functions like Euclidean distance, Mean Square Error for measurement of similarity.

\section{RELATED WORK}

Existing CBIR systems are based on color, texture, shape. In color based based approach color is extracted from the images to form feature vectors most. Color is represented using common color spaces models like sRGB, Adobe RGB and ProPhoto RGB, CMYK, HSV, HSL then similarity measure is computed using Euclidean distance. Texture describes statistical properties of an image; hence two images can be compared based on Texture. There are various region-based and boundary based techniques available for retrieval using shape. Swain and Ballard [3] proposed idea to use color histogram for retrieval. Lin et al. [4] proposed a scheme based on color-texture and color-histogram. Hiremath and Pujari [5] came up with a CBIR technique based on color, shape and texture. Rao et al. [6] proposed dominant color based retrieval system. CBIR has been used in many applications like recognition systems, tumor detection, detecting and blocking adult content.

\section{PROPOSED METHODOLOGY}

The proposed model is shown in Figure 2.

\subsection{Crawling}

Images are crawled using a basic image crawler and fed for color extraction. Crawled images are re-sampled to 1 pixel using average weight algorithm [7].

\subsection{Color Extraction}

Color of pixel is represented in Red Green Blue (RGB) color space and (Hue Saturation Lightness) HSL color space [8]. We used two color spaces for better human perception of color.

In Hue Saturation Lightness (HSL) color space, Hue represents the color, Saturation how much color is present, hue the brightness or darkness in color.

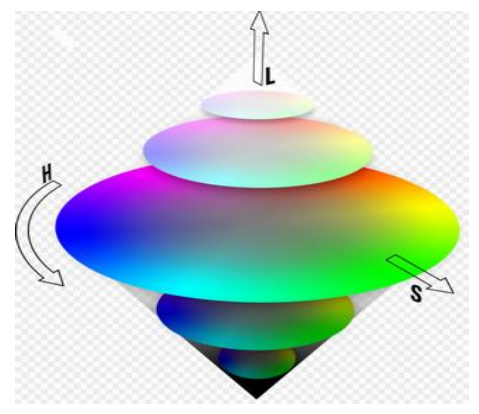

Fig 1: HSL color space 


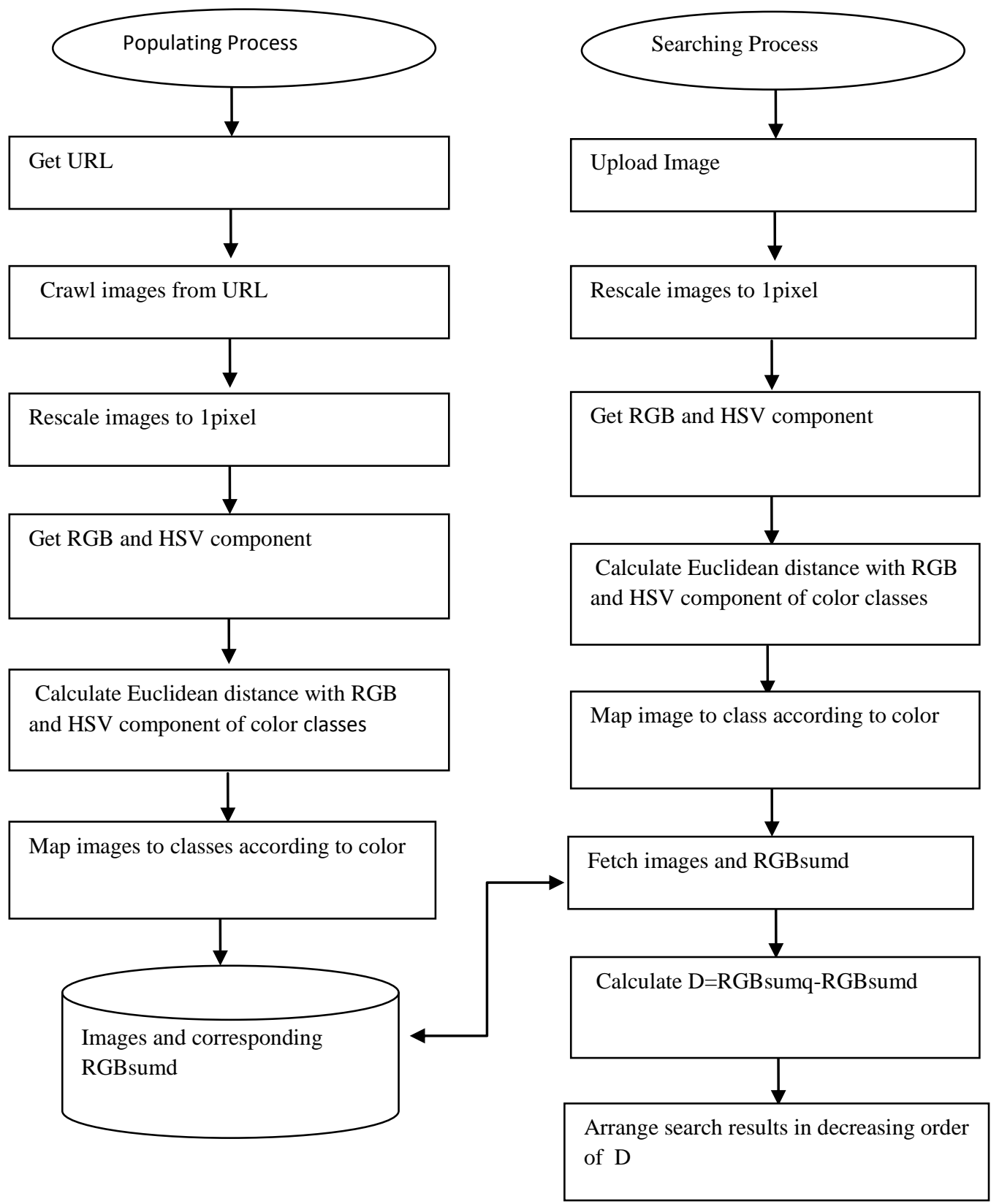

Fig 2: Flow chart of proposed system

In Red Green Blue (RGB) color space-There are three channels in RGB color space. Red channel (Redness), Green channel (Greenness), Blue channel (blueness) mixing these colors allow us to generate any color.

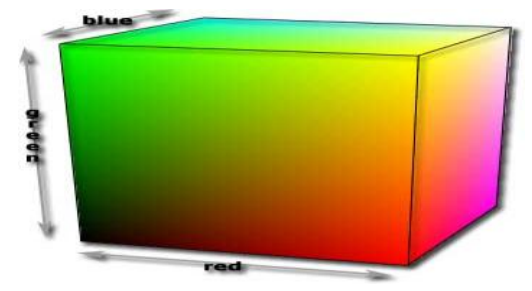

Fig 3: RGB color space

\subsection{Euclidean Distance}

Images crawled are mapped to colors after color extraction from image using Euclidean distance. Suppose an image $\mathrm{X}$ is crawled. $\mathrm{X}$ is first re-sampled to 1pixel. Image $\mathrm{X}$ is mapped to color $\mathrm{Y}$ if Euclidean distance is minimum for Y. Euclidean distance for RGB and HSL color space is given by

$$
\begin{aligned}
& \mathrm{D} 1=\left(\mathrm{X}_{\mathrm{R}}-\mathrm{Y}_{\mathrm{R}}\right)^{2}+\left(\mathrm{X}_{\mathrm{G}}-\mathrm{Y}_{\mathrm{G}}\right)^{2}+\left(\mathrm{X}_{\mathrm{B}}-\mathrm{Y}_{\mathrm{B}}\right)^{2} \\
& \mathrm{D} 2=\left(\mathrm{X}_{\mathrm{H}}-\mathrm{Y}_{\mathrm{H}}\right)^{2}+\left(\mathrm{X}_{\mathrm{S}}-\mathrm{Y}_{\mathrm{S}}\right)^{2}+\left(\mathrm{X}_{\mathrm{L}}-\mathrm{Y}_{\mathrm{L}}\right)^{2}
\end{aligned}
$$

\subsection{Matching Process}

Color Feature of the image given as query are calculated in same way as for crawled images Euclidean distance of query image with 1600 colors is calculated, now search is performed 
only in the table corresponding to particular color with minimum Euclidean distance. The difference between RGBsum of queried image and RGBsum of images present in table is calculated and results are shown in decreasing order of difference in RGBsum.

\section{RESULTS}

We have used a data set of 10,000 images of different colors. System is implemented on intel atom processor with $2 \mathrm{~GB}$ RAM using PHP. We can see efficiency of using this method.

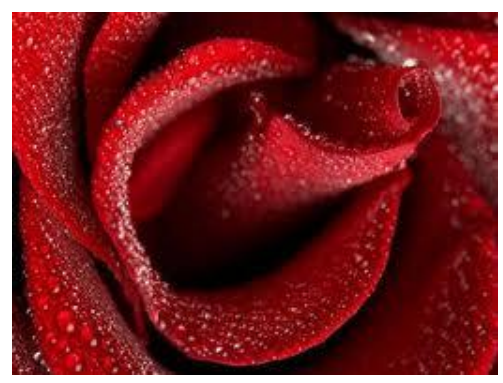

Fig 4: Query image

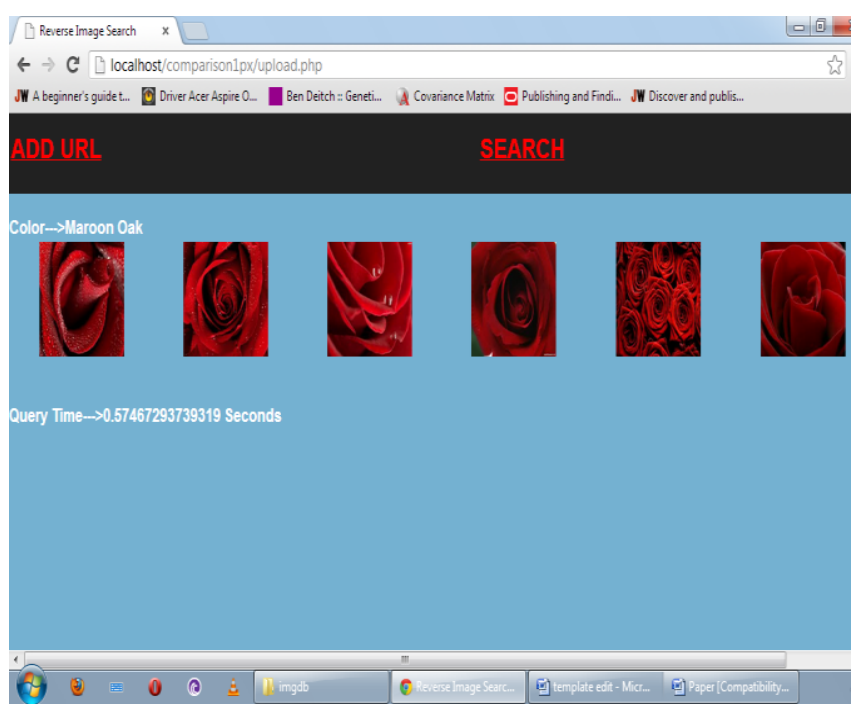

Fig 5: Search results

\section{CONCLUSION}

Color is main feature present in multimedia. This paper focuses only on efficient retrieval based on color. Proposed method uses color spaces and Euclidean distance. Further, using color based classification makes retrieval process very fast. CBIR based on color has been used in various applications like detecting skin lesions, retrieving tattoo images etc.
Results show that the efficiency by processing a single pixel using this method is great. Further, if method is used for large no of pixels it can produce great results with very high accuracy and integrating other features like texture, shape will provide best results.

\section{ACKNOWLEDGMENTS}

Harsh Kumar Sarohi would like to thank Prof. Niraj Singhal, School of Computer Engineering \& Information Technology, Shobhit University whose knowledge and encouragement helped in the pre research period.

\section{REFERENCES}

[1] T. Sumathi, C. Lakshmi Devasena, M. Hemlata, "An Overview of Automated Image Annotation Approaches", International Journal of Research and Reviews in Information Sciences, Vol. 1, March 2011.

[2] A. Vadivel, A. K. Majumdar, S. Sural, "Characteristics of Weighted Feature Vector In Content-Based Image Retrieval Applications", Proceedings of Intelligent Sensing and Information Processing, 2004, ICISIP, 2004.

[3] M. Swain, D. Ballard, "Color indexing", International Journal of Computer Vision, 1991.

[4] C.H. Lin, R.T. Chen and Y.K. Chan, "A smart contentbased image retrieval system based on color and texture feature", International Journal of Computer Vision, 1991.

[5] P. S. Hiremath, J. Pujari, "Content Based Image Retrieval based on Color, Texture, Shape features using Image and its complement", $15^{\text {th }}$ International Conference on Advance Computing and Communications. IEEE, 2007.

[6] M.B. Rao, B.P Rao, A. Govardhan, "CTDCIRS: Content based Image Retrieval System based on Dominant Color and Texture Features", International Journal of Computer Applications, Vol. 18, No.6, pp-0975-8887, 2011.

[7] P. B. Thawari, N. J. Janwe, "CBIR Based On Color And Texture", International Journal of Information Technology and Knowledge Management, Vol. 4, No. 1, pp. 129-132, 2011.

[8] Manimala Singha, K. Hemchandran, "Performance analysis of Color Spaces Image Retrieval”, Assam University Journal of Science \& Technology: Physical Sciences and Technology, Vol. 4, 2011. 